\title{
LA ESCUELA PRIMARIA RURAL EN ITALIA ENTRE LOS SIGLOS XIX Y XX
}

\section{The rural school in Italy between the 19th and the 20th century}

\section{Luca Montecchi*}

Fecha de recepción: 03/04/2017 • Fecha de aceptación: 18/06/2017

Resumen. El artículo tiene como objetivo explicar el desarrollo histórico de la educación primaria impartida a los jóvenes campesinos italianos entre la Unificación nacional y la caída del Fascismo (1861-1943). En particular, pretende destacar las continuidades y los cambios en las políticas escolares promulgadas por el Gobierno Italiano en relación con las escuelas rurales, analizando — desde un punto de vista institucional— la evolución de la legislación y la historia de las instituciones estatales y privadas que fueron encargadas de organizar las escuelas para los jóvenes campesinos entre los siglos XIX y XX.

Palabras clave: Analfabetismo; Escuela primaria; Siglos XIX-XX; Mundo campesino, Italia.

Abstract. The aim of this work is to trace the historical events surrounding primary education for Italian children between the Unification of Italy and the fall of Fascism (1861-1943). We focus particularly on the continuity and the changes in school policy promoted by the Italian government in relation to rural schools, offering an analysis, from an institutional point of view, of the evolution of the legislation and of the history of the state and private institutions in charge of organizing schools for young farmers between the half of the 19th and of the 20th century.

Keywords: Illiteracy; Elementary school; 19th-20th centuries; Countryside, Italy.

\footnotetext{
" Dipartimento di Scienze della Formazione, dei Beni Culturali e del Turismo, Università degli Studi di Macerata. Ple Luigi Bertelli, 1 - C.da Vallebona, 62100 Macerata, Italia. 1.montechi@ libero.it
}

Cómo citar este artículo: Montecchi, Luca. «La escuela primaria rural en Italia entre los siglos XIX y XX». Historia y Memoria de la Educación 7 (2018): 81-106. 


\section{INICIANDO}

Cuando Italia llegó a la cita con la unificación nacional en 1861, la mayoría de la población vivía en el campo y en las zonas de montaña. Italia era un país predominantemente agrícola, tanto es así que el porcentaje de la población rural ascendía a un $74,5 \%$ y así se mantuvo durante mucho tiempo. ${ }^{1}$ Un estudio estadístico da cuenta, de hecho, de que el número de personas que vivía en las zonas rurales en 1960 era del 53,5\%. ${ }^{2}$ Estas pocas cifras son suficientes para entender el papel central en la historia italiana del campo - de las zonas rurales-y de las condiciones de vida de aquellos que en él vivían a la hora de trazar un perfil histórico de la Italia contemporánea.

En cuanto a la investigación histórico-educativa, la importancia de reconstruir de forma orgánica y exhaustiva cómo respondió la clase dirigente a las necesidades educativas de los campesinos es una adquisición reciente. ${ }^{3}$ Durante mucho tiempo, de hecho, los problemas de la difusión del conocimiento en las zonas rurales se consideraron de modo conjunto con las cuestiones relacionadas con la difusión de las escuelas entre las clases trabajadoras de las ciudades. La historiografía, de hecho, solo ha utilizado desde los años cincuenta la categoría global de «instrucción popular», es decir, una expresión que incluía los problemas relacionados tanto con la educación en las zonas rurales como en las urbanas. ${ }^{4} \mathrm{Si}$ este punto de vista ha tenido la ventaja de ofrecer una visión de conjunto, no ha permitido, sin embargo, apreciar plenamente las diferencias significativas que aun así existían entre la "escuela rural» y la "escuela urbana», como, para empezar, tomar la decisión —adoptada por la clase dirigente por medio de la Ley Casati de 1859- de utilizar deliberadamente dos denominaciones diferentes para indicar la escuela de educación primaria rural y la escuela de educación primaria urbana. No se trató solo de una

\footnotetext{
${ }^{1}$ Los datos se toman de la Tabla 2.5 publicada en Fernanda Spagnoli, «Popolazione urbana e rurale», Annali di statistica, 17 (1965): 181.

${ }^{2}$ Los datos se toman de la Tabla 2.8 publicada en Fernanda Spagnoli, «Popolazione urbana e rurale», 187.

${ }^{3}$ Luca Montecchi, I contadini a scuola. La scuola rurale in Italia dall'Unità alla caduta del fascismo (Macerata: Eum, 2015).

${ }^{4}$ Larga sería la lista de trabajos que han utilizado la categoría de la «educación popular» a partir del estudio pionero de Dina Bertoni Jovine (Storia della scuola popolare in Italia, 1953), que la utilizó por primera vez.
} 
cuestión léxica o jurídica: la escuela rural en Italia fue una realidad con su propia especificidad que merece ser estudiada en su conjunto.

\section{DE LA LEY CASATI (1859) AL TIEMPO DE GIOLITTI}

Fue la Ley Casati, aprobada el 13 de noviembre de 1859 en el Reino de Cerdeña y luego extendida a las provincias italianas incorporadas a la monarquía de los Saboya, la que creó en Italia la «escuela rural». Esto no significa que antes de esa fecha no existieran algunas escuelas que proporcionaran los rudimentos básicos del conocimiento en las áreas rurales más desarrolladas de la península —donde las comunidades podían disponer de los recursos económicos necesarios para pagar al maestro, que la mayoría de las veces era el cura-, pero casi siempre quienes asistían no eran los hijos de los campesinos, sino los hijos de aquellas familias que formaban la pequeña burguesía que vivía en las áreas rurales (pequeños terratenientes, artesanos, médicos, farmacéuticos, etc.).

Como se ha anticipado, la Ley Casati fundaba la escuela de educación primaria, encargando su gestión a los ayuntamientos y subdividiéndola en dos categorías: la «escuela urbana» y la «escuela rural». A su vez, estas estaban divididas en tres «clases» de acuerdo con el tamaño de la población residente en el municipio: eran urbanas de primera, segunda y tercera clase las establecidas en localidades donde la población era de más de 40.000, 15.000 y 4.000 habitantes respectivamente, y eran rurales de primera, segunda y tercera clase las establecidas en localidades con una población que excedía respectivamente de los 3.000, 2.000 y 500 habitantes respectivamente. ${ }^{5}$ La educación primaria constaba de dos etapas (etapa inferior y etapa superior, ambas con una duración bienal), pero solo la inferior era obligatoria en todos los municipios y también en las fracciones municipales —entidades locales menores-con al menos 50 niños susceptibles de asistir a la escuela. La segunda etapa era obligatoria solo en municipios con más de 4.000 habitantes.

Si bien la creación de un número considerable de escuelas de educación primaria en muchos centros habitados del recién nacido Reino de Italia tuvo sin duda algún resultado positivo que otro, durante los años

\footnotetext{
${ }^{5}$ Giuseppe Inzerillo, Storia della politica scolastica in Italia. Da Casati a Gentile (Roma: Riuniti, 1974), 83.
} 
setenta resultaron patentes las limitaciones inherentes a la legislación introducida por Casati. La primera, y la más interesante a efectos de esta discusión, fue la de haber tomado como único criterio para distinguir la escuela rural de la urbana el número de estudiantes que a ellas asistirían: de esta forma, el título de escuela rural también se atribuía a escuelas que surgían no necesariamente en el campo, desalentando, en la práctica, el nacimiento de una escuela que respondiera a las necesidades educativas reales de las poblaciones campesinas, ya que permanecía ajena al contexto socioeconómico en el que se encontraba. Una segunda limitación inherente a la Ley Casati era la representada por los modestos objetivos que pretendía en cuanto a la lucha contra el analfabetismo y a la mejora del nivel intelectual y moral de los campesinos. De hecho, la etapa inferior de educación primaria, con una duración de solo dos años, no proporcionaba ciertamente un nivel suficiente y adecuado de alfabetización. Una tercera limitación de la Ley Casati fue la de haber confiado la fundación y la gestión de las escuelas de educación primaria a los ayuntamientos. De este modo, el Estado mostraba su falta de interés por el problema de la alfabetización de las clases populares, endosando una pesada responsabilidad a los ayuntamientos, la mayoría de los cuales carecían de los recursos económicos para establecer escuelas en todos los lugares donde había una necesidad real.

Con la llegada al poder de la izquierda, se aprobaba la Ley Coppino (1877) que trataba de hacer más eficaz la obligación de escolarización, que precisamente en el campo era mayormente incumplida por muchas familias campesinas que no comprendían la utilidad de la educación escolar. La nueva ley establecía que los niños, después de haber completado la etapa inferior de educación primaria, tenían que asistir durante un año a las escuelas nocturnas en los municipios en los que estas estaban operativas o a las escuelas festivas femeninas. Estas últimas también se llamaban «escuelas complementarias», y su objetivo era el de "continuar y ampliar la enseñanza de las asignaturas exigidas como obligatorias por el art. 2» de la Ley Coppino. A pesar de estas intenciones, las normas de 1877 en esta área demostraron ser «muy pronto insuficientes» y tampoco llevó a un resultado positivo la legislación posterior.

Mientras tanto, en los años ochenta de ese mismo siglo el destino de las escuelas rurales se cruzaba con un debate mucho más amplio sobre la 
necesidad de transferir la gestión de las escuelas de educación primaria de los ayuntamientos al Estado: solo de esta manera se creía poder proporcionar más recursos económicos a la escuela de educación primaria italiana en general y a los enseñantes en particular. A pesar de algunas campañas de prensa lanzadas por los periódicos magisteriales de la época, las peticiones de nacionalización de toda la educación primaria cayeron en saco roto y habrá que esperar más de veinte años antes de que este objetivo se logre con la aprobación de la Ley Daneo-Credaro (1911). La temporada de reformas que precedió a la puesta en marcha de esta importante ley se caracterizó por señales que ponían de manifiesto una intención muy distinta por parte de la clase política. La crisis económica y política de final de siglo alarmó seriamente a la clase dirigente italiana que empezó a temer el final de un orden social que hasta entonces se había considerado eterno. El símbolo de esta nueva sensibilidad fueron los nuevos programas para las escuelas de educación primaria (1894) aprobados por el ministerio de Guido Baccelli. Estos nacían a raíz de ese clima de involución política y cultural que caracterizó a los últimos quince años del siglo, dominados por las presiones nacionalistas y por la explosión de tensiones sociales por mucho tiempo soterradas. ${ }^{6}$ Significativamente, los programas de 1894 se inspiraban en el deseo de ofrecer a las nuevas generaciones unos pocos conceptos básicos útiles para poderse integrar en el mundo laboral y en la comunidad civil y política, de acuerdo con la fórmula de «enseñar al pueblo lo suficiente, educarlo todo lo que se pueda». Desde este punto de vista, adquiría un significado concreto la introducción en las escuelas de educación primaria de la enseñanza de la llamada «cultura material», considerada necesaria para poder favorecer el desarrollo económico del país y la modernización de la agricultura y, asimismo, disciplinar a las clases populares que amenazaban con mostrarse sensibles a la propaganda socialista y radical. ${ }^{7}$ Esto se tradujo, a nivel didáctico, en la introducción en las escuelas de educación primaria del trabajo manual educativo, por una parte, y, por otra, de los estudios agrícolas, a raíz de una convicción que ganó cada

\footnotetext{
${ }^{6}$ Sobre la crisis de fin del siglo, la bibliografía es bastante extensa. Sólo queremos recordar: Umberto Levra, Il colpo di stato della borghesia. La crisi politica di fine secolo e l'età giolittiana (Torino: UTET, 1982).

${ }^{7}$ Gaetano Bonetta, ««Insegnare la cultura materiale». Istruzione agraria e lavoro manuale nell'Italia del XIX secolo», en Storia delle Istituzioni educative in Italia tra Otto e Novecento, ed. Lucia Romaniello (Milano: Amici del Museo del Risorgimento, 1996), 153-164.
} 
vez más credibilidad en los años ochenta y noventa según la cual, como escribió el pedagogo Pietro Pasquali, la escuela había «exagerado la ocupación intelectual en detrimento de las facultades de uso cotidiano en la vida». ${ }^{8}$ Con el fin de alcanzar estos objetivos, Baccelli manifestaba, ya con los programas de 1894, la esperanza de que a los maestros se les concediera un pequeño huerto, situado posiblemente cerca de la escuela, para poder utilizarlo con fines educativos. Fue, sin embargo, por medio de la circular del 20 de julio de 1898, que Baccelli decidió introducir, aunque de forma opcional, la enseñanza práctica de las primeras nociones de agricultura en las escuelas rurales y dar un nuevo impulso al proyecto para la creación de los huertos escolares. ${ }^{9}$

Superada la crisis de final del siglo, se abría en Italia un período de reformas de carácter liberal que tenían como objetivo la integración de estratos cada vez más amplios de la población en la vida política del país y, tal y como muchos esperaban, la prevención de futuros disturbios y alteraciones sociales y el crecimiento de los consensos hacia las fuerzas de la oposición. ${ }^{10}$ La primera intervención desde este punto de vista fue la Ley Orlando (1904) que amplió a los doce años de edad la duración de la educación obligatoria con la creación de la etapa popular de duración bienal, que se añadía a los cuatro años de las etapas de educación primaria inferior y superior; pero este ajuste era más formal que sustancial, ya que se limitaba a los cursos de primaria existentes en el municipio. Los beneficiarios eran sobre todo los niños de la ciudad, donde a veces existía la etapa superior, no ciertamente en el campo donde la instrucción se limitaba a los tres primeros cursos.

Mucho más importante, sin embargo, fue la Ley 383 del 15 de julio de 1906, que contenía «medidas para las provincias meridionales, para Sicilia y para Cerdeña». De hecho, en ella se preveía la posibilidad de establecer escuelas de educación primaria inferior de tercera clase rural completamente a cargo del Estado en las fracciones municipales o aldeas con al menos 40 alumnos de inscripción obligada, condiciones que era

\footnotetext{
${ }^{8}$ Bonetta, «Insegnare la cultura materiale», 160.

${ }^{9}$ Enzo Catarsi, Storia dei programmi della scuola elementare, 1860-1985 (Firenze: La Nuova Italia, 1990), 51-54.

${ }^{10}$ Ester De Fort, La scuola elementare dall'Unità alla caduta del fascismo (Milano Il Mulino, 1996), 199-204.
} 
más común encontrar en el campo y en las zonas de colinas y montaña. Se trataba de una verdadera revolución, que anticipó la avocación de las escuelas por el Estado en 1911, dado que por primera vez se afirmaba el principio de la intervención directa del Estado en la creación de escuelas rurales allí donde hubiere más necesidad. La ley de 1906 preveía, además, que también las escuelas de educación primaria inferior opcionales, mantenidas por los ayuntamientos, pudieran clasificarse como de tercera rural; a cambio, el Estado se encargaría del gasto necesario para el aumento del salario del enseñante. También se establecía en el ministerio una Commissione Centrale per la diffusione dell'istruzione elementare nel Mezzogiorno e nelle isole. Su tarea sería la de administrar eventuales fondos residuales derivados de la concesión de los fondos asignados como resultado de esta ley, asesorar al ministro y señalar si un ayuntamiento había incumplido sus obligaciones educativas. En este caso, el superintendente podía enviar a ese ayuntamiento un responsable didáctico para hacerse cargo de los servicios escolares con las mismas competencias que un comisario de la prefectura. El artículo 77 preveía, por último, que estas medidas afectarían no solo a las regiones meridionales y a las islas, sino también a una gran parte del centro de Italia.

La ley de 1906 fue la que marcó un verdadero punto de inflexión para la escuela rural después de cincuenta años de estancamiento sustancial que caracterizó al período que había comenzado con la promulgación de la Ley Casati. De hecho, durante el curso 1907-1908 se establecieron 1.782 nuevas escuelas rurales de tercera clase en su totalidad a cargo del Estado y 1.462 escuelas debido a las subdivisiones. Además, 333 escuelas habían sido transformadas de opcionales a clasificadas. Haciendo balance a 1 de enero de 1908, nada menos que 3.577 escuelas habían sido creadas por la Ley del Mediodía de 1906. ${ }^{11}$ Números que eran enfáticamente subrayados por el director general de Educación Primaria y Popular, Camillo Corradini, en su famosa Investigación sobre el estado de la escuela de educación primaria en el Reino, publicada en 1910 y destinada a convertirse, para una parte de la clase dirigente, en objeto de reflexión sobre las condiciones en las que se encontraban las escuelas de educación primaria.

${ }^{11}$ Camillo Corradini, Relazione presentata a S. E. il Ministro della Pubblica Istruzione dal Direttore Generale per la Istruzione primaria e popolare, (Roma: tip. Operaia Romana Cooperativa, 1910), 40. 
Otro momento decisivo en la historia de las escuelas rurales está representado por la Ley Daneo-Credaro (1911), que constituyó el mayor esfuerzo realizado desde la Unificación para difundir la educación primaria en el país. Elaborada por el ministro Luigi Credaro, que incorporó algunas modificaciones a un proyecto de ley presentado por su predecesor Edoardo Daneo, marcó un punto de inflexión porque establecía la tan solicitada transición de la gestión de la enseñanza primaria de los ayuntamientos al Estado. La medida afectó a todas las escuelas de educación primaria, tanto las urbanas, como las rurales, excepto en los municipios capitales de provincia y de circunscripción — subdivisión administrativa de los antiguos reinos de Cerdeña y de Italia-. En cuanto a las escuelas rurales con clases agrupadas bajo un único maestro y con un único horario, creadas en los municipios y en las aldeas, la ley preveía su reorganización de la siguiente manera: en aquellos municipios y aldeas donde se había establecido solo una de estas escuelas, al maestro se le encomendaba la enseñanza, en diferentes horarios, del primer al tercer curso.

\section{DEL ENTE CONTRA EL ANALFABETISMO EN LOS ADULTOS A LA OBRA CONTRA EL ANALFABETISMO: DOS INTENTOS DE REFORMA}

La entrada de Italia en la Gran Guerra, que se produjo en 1915, detuvo todo intento reformador. Hubo que esperar hasta la posguerra para ver reabrirse el debate sobre la reforma escolar. De hecho, más allá de las inevitables diferencias políticas y diferentes sensibilidades ideológicas, todos en general estimaban oportuna la necesidad de rediseñar el sistema educativo italiano, tratando, entre otras cosas, de introducir medidas de política educativa de cara a la reducción del analfabetismo creciente. La trágica experiencia de la guerra había demostrado que — por decirlo en palabras de un liberal, así como promotor de una original forma de escuelas rurales, como era el terrateniente y senador Eugenio Faina-, «un pueblo vale por lo que sabe», y que las debilidades mostradas por Italia durante el conflicto debían atribuirse también a la ignorancia, que a menudo se convertía en ineptitud, de las masas populares, especialmente las campesinas. En los círculos liberales más abiertos al cambio, este sentimiento estaba ampliamente generalizado, aunque con diferentes 
objetivos, entre aquellas personalidades del mundo de la cultura — tanto socialistas como católicas- que deseaban la llegada de una escuela inspirada en fuertes ideales nacionales y patrióticos. ${ }^{12}$

El primer sector en el que se decidió intervenir, incluso antes del de la lucha contra el analfabetismo de los niños, fue en el de la lucha contra el analfabetismo de los adultos, con la esperanza de obtener más fácilmente algunos resultados positivos. Entre finales de 1918 y principios de 1919 fue Corradini, el autor del conocido Estudio sobre la escuela primaria italiana (Inchiesta sulla scuola elementare italiana), quien planteó la idea de hacer frente a esta emergencia con una intervención igualmente extraordinaria, ubicada fuera de la organización escolar estatal, capaz de resolver el problema «de la manera más práctica y expedita»o, como se dijo entonces, "al estilo americano» $\mathrm{o}$ "al estilo de Garibaldi». ${ }^{13} \mathrm{Su}$ propuesta se refería, de hecho, a la creación de una entidad específica contra el analfabetismo de los adultos, autorizada a organizar breves cursos de alfabetización para personas de entre 15 y 50 años. La idea de Corradini, presentada a los líderes de la UIEP o Unión Italiana de Educación Popular (Unione Italiana dell'Educazione Popolare), fue aceptada de inmediato y se formó un comité formado por un grupo de parlamentarios de diferente orientación política y por representantes del mundo magisterial y cultural.

Con tales premisas, se llegaba así al 9 y 10 de marzo de 1919, año en que se celebró en Roma el congreso que llevaba por título Las necesidades más urgentes de la enseñanza y de la educación popular, destinado a convertirse, sin duda alguna, en el principal punto de unión del vasto movimiento de reforma de la escuela rural. ${ }^{14}$ De hecho, a pesar de la violenta lucha política que caracterizó a la inmediata posguerra italiana, en el ámbito de la educación popular se podía apreciar una inusual convergencia entre los principales partidos que no evitó, sin embargo, atraer las sospechas y las críticas de algunos sectores la izquierda más extrema ante la actitud de cooperación mostrada por Filippo Turati con respecto a personalidades con una cultura liberal y conservadora. Sospechas

${ }^{12}$ Giorgio Chiosso, L'educazione nazionale da Giolitti al primo dopoguerra (Brescia: La Scuola, 1983).

${ }^{13}$ Camillo Corradini, «Una crociata contro l'analfabetismo degli adulti», I diritti della scuola, 9 (10 de enero de 1919): 140-141.

14 «I lavori del convegno», La coltura popolare, 3-4, (marzo-abril de 1919): 178-285. 
políticas aparte, el congreso abordó la cuestión de la escuela rural en un animado debate en el que se pusieron de manifiesto dos visiones opuestas sobre el concepto mismo de escuela rural: por un lado, los defensores de una escuela que no perdiera el carácter humanista; por otro, los partidarios de una escuela que diera prioridad a la formación profesional del campesino. Al final fue aprobado un documento que, en esencia, se adhería a la tesis de una escuela rural que salvaguardara el aspecto de la formación humana del niño y que no estuviera demasiado desequilibrada por lo que respectaba a la profesionalización.

El apoyo político de un gran número de parlamentarios tuvo el efecto de llevar a la práctica los proyectos desarrollados en el congreso romano de la primavera de 1919. De hecho, pocos meses después, el 2 de septiembre, bajo el control del ministerio de Agostino Berenini, se establecía el Ente para la Instrucción de Adultos Analfabetos (Ente per l'Istruzione degli Adulti Analfabeti), un organismo en el que el Estado delegaría la tarea de organizar las escuelas festivas y nocturnas de la Italia centro-meridional. La importancia de esta organización era doble, ya que se le puso a cargo no solo de la educación de los adultos, sino también del refuerzo de la educación primaria para niños. ${ }^{15} \mathrm{~A}$ pesar de que nació gracias al encuentro entre sensibilidades diferentes pero unidas por el deseo común de erradicar el mal del analfabetismo, la nueva organización, sin embargo, tendría que lidiar con una serie de dificultades que primero ralentizaron el inicio de las actividades y luego decretaron su supresión en unos pocos meses. Hay que tener en cuenta, de hecho, que un par de semanas después hubo elecciones generales para la renovación de la Cámara, las primeras después del final de la guerra, un hecho que había sumido al país en un fuerte choque entre los partidos. El nuevo escenario político, que había visto la consolidación del Partido Popular Italiano (PPI) como una fuerza indispensable para la formación de cualquier gobierno, no permitió un feliz comienzo de las actividades del recién nacido organismo. Una serie de ajustes en el decreto aportados por el gobierno para satisfacer algunas exigencias del PPI, llevaron a las barricadas a la oposición socialista, empezando por Turati y Matteotti. Después de la presentación de una pregunta parlamentaria, que tuvo el efecto de denunciar el intento de una demostración de fuerza por parte del gobierno, se reunió

15 «All'Ente contro l'analfabetismo», I diritti della scuola, 15 (1 de febrero de 1920): 234. 
el consejo de la organización. Tras de un tormentoso debate, algunos de sus miembros, incluido el mismo Turati, presentaron su dimisión.

Encallado en los bajíos de la política parlamentaria, la organización asistía a su declive al que no pudo poner remedio ni siquiera el despido de Baccelli y el nombramiento como nuevo ministro de educación de Andrea Torre, elegido por Nitti en el contexto de su segundo gabinete puesto en marcha en mayo de 1920. De hecho, el 16 de junio, después de ni tan siquiera un mes, el segundo gobierno de Nitti caía dando paso al regreso de Giolitti. El nuevo ministro, el distinguido filósofo Benedetto Croce - en mayor medida atento a otras cuestiones, como las oposiciones-, decidía la supresión de la desafortunada organización con el Decreto del 5 de agosto de 1920. Esta decisión no convenció, sin embargo, a la oposición socialista, que la consideró una vacilación del gobierno que se movía aún con incertidumbre en esta área.

Por su parte, Croce dio a entender que en la base de esta elección había un cambio en la estrategia: a partir de entonces la lucha contra el analfabetismo se llevaría a cabo en primer lugar entre las nuevas generaciones y, en menor medida, entre los adultos, dando prioridad a la escuela de educación primaria. A este respecto, afirmó que establecería un tramo inicial de 2.000 nuevas escuelas de educación primaria, que se sumarían a las aproximadamente 78.000 ya existentes, con el ambicioso objetivo de llegar en un futuro a las 100.000. ${ }^{16}$ Para Matteotti se trataba, sin embargo, de un número insuficiente para satisfacer las necesidades reales de la escuela italiana, como declaró en una de sus interpelaciones debatida en la Cámara el 22 de noviembre de 1920. Eran preocupaciones comprensibles si se piensa que el propio Croce tuvo que rescatar poco después la idea de encomendar a una adecuada entidad la organización de las escuelas para analfabetos, en lugar de pensar en un compromiso directo y total del Estado a este respecto. El filósofo no tuvo tiempo para decretar el nacimiento del nuevo organismo debido a la crisis de gabinete Giolitti, que llevó a la formación del gobierno Bonomi, pero fue su sucesor, Orso Mario Corbino, quien concretó la creación de tal organismo, firmando el 28 de agosto de 1921 el decreto que establecía el organismo Obra contra el Analfabetismo (Opera contro l'Analfabetismo). Este último comenzaba su actividad justo a tiempo para el inicio del año escolar

${ }^{16}$ Bollettino Ufficiale del Ministero della Pubblica Istruzione, 35 (26 agosto 1920): 1514. 
1921-1922. Pero, a diferencia del experimento llevado a cabo en 1919, esta vez el nuevo organismo era capaz de funcionar plenamente gracias a la convergencia política que había surgido, y a la creación y comprensión del hecho de que ya no era posible otro fracaso.

La nueva entidad se presentaba como un organismo a medio camino entre el Estado y las asociaciones privadas. El primero se eximía de una pesada carga - que no habría podido soportar sobre sus hombros-con el establecimiento de unas pocas miles de escuelas públicas para niños y adultos; al mismo tiempo, el Estado, cediendo a asociaciones privadas la tarea de la efectiva dirección de las escuelas, no renunciaba a desempeñar una acción de dirección y coordinación, ni tampoco rehusaba cumplir la función educativa, que era considerada por un amplio grupo de personas —considérense los socialistas, pero también los idealistas- una prerrogativa de absoluta incumbencia del Estado. Las asociaciones seleccionadas a este respecto fueron cuatro, todas ya existentes y que operaban en el campo de la educación popular a través de la gestión de jardines de infancia, clases para inmigrantes y escuelas rurales. Se trataba de las Escuelas para los Campesinos del Agro Romano (Scuole per i Contadini dell'Agro Romano), la Asociación Nacional para los Intereses del Mediodía (Associazione Nazionale per gli Interessi del Mezzogiorno), de ahora en adelante llamada Animi, la Sociedad Humanitaria (Societá Umanitaria) y el Consorcio Nacional de Emigración y Trabajo (Consorzio Nazionale Emigrazione e Lavoro). Además, hay que subrayar que la identificación de las cuatro asociaciones se hizo también siguiendo un criterio político, con el objetivo de involucrar en este proyecto a tantas tendencias y directrices ideológicas como fuera posible. Las dos primeras asociaciones eran, de hecho, una expresión de algunos sectores del liberalismo, la tercera de los ambientes socialistas reformistas milaneses que tenían a Turati como representante, mientras que la cuarta rendía cuentas al partido popular.

Según lo establecido por la ley, la actividad de la entidad seguía tres direcciones: creación de escuelas diurnas para hijos de agricultores, pastores, pescadores, mineros y jornaleros, que debían establecerse «en el campo y en canteras de construcción y fábricas» donde pudieran agruparse por lo menos 20 alumnos de entre 6 a 14 años con un horario de cinco horas diarias para un curso de 180 horas; creación de escuelas nocturnas para trabajadores mayores de 12 años en los centros en los 
que fuera posible reunir al menos 15 estudiantes con horario de por lo menos dos horas diarias de un curso de lecciones suficientes para llevar a cabo un programa didáctico que en el primer curso llevara al analfabeto a saber leer y escribir; creación de escuelas festivas que se establecerían en pequeños centros con un curso básico, especialmente para mujeres, con nociones útiles para la vida profesional (industrial y agrícola), y con el uso de diversos medios como el cine, las experiencias prácticas, las lecturas y las conferencias. Asimismo, se estableció que el programa didáctico para las escuelas diurnas y para las escuelas nocturnas fuera el mismo que el de la etapa inferior de educación primaria, y que el progreso de los alumnos tenía que ser verificado mediante exámenes. Por otra parte, en cuanto a la elección del profesorado, se había previsto que los maestros de las escuelas serían elegidos por cada una de las asociaciones delegadas y, si estos poseían el diploma de habilitación, verían reconocido como válido su servicio a efectos de su carrera magisterial.

En los dos primeros años de actividad, la acción de la Obra se dedicó especialmente a la instrucción de adultos. De hecho, en 1921-1922 funcionaron 2.057 escuelas nocturnas y 319 escuelas festivas para adultos y 213 escuelas diurnas para niños, números destinados a crecer durante el siguiente año, en el que se activaron 3.187 entre escuelas nocturnas y festivas y 403 escuelas diurnas, de las cuales, sin embargo, un centenar ya existían, porque fueron establecidas autónomamente por la entidad Escuelas para los Campesinos del Agro Romano. Sin embargo, vistos con la debida distancia los resultados obtenidos, sin menospreciar el trabajo realizado por estas entidades, no se puede evitar notar como el inicio de la actividad de la Obra estuvo marcado por una serie de puntos críticos, empezando por esas limitaciones financieras que pusieron de relieve en breve tiempo cómo la sordera generalizada de muchos sectores de la clase dirigente hacia los problemas de la difusión de la cultura y del conocimiento entre los jóvenes campesinos ciertamente no había desaparecido.

Lo dicho hasta ahora sobre la Obra contra el Analfabetismo no debe hacernos olvidar el hecho de que esta funcionó durante el bienio 1921-23 solo en los centros habitados de la Italia centro-meridional donde el número de obligados a la asistencia era inferior a las cuarenta unidades. Esto significaba que en todas las zonas rurales de las regiones septentrionales, y en parte de las del centro meridional, siguió vigente la anterior 
legislación que preveía la adjudicación de la gestión de las escuelas rurales directamente al Consejo Provincial de Educación.

\section{LA ESCUELA RURAL DURANTE EL PERÍODO FASCISTA (1922-1943)}

Con la subida al poder del fascismo, que tuvo lugar a finales de octubre de 1922, se abría una nueva fase en la historia de la educación rural, que culminaría con el exitoso intento del Régimen por apoderarse de estas escuelas y doblegar sus enseñanzas en clave ideológica y política. Sin embargo, los primeros pasos realizados por el nuevo gobierno abogaban por una continuidad sustancial de cuanto había sido hecho en los últimos años. De hecho, el nuevo ministro, el filósofo idealista Giovanni Gentile, expresó desde el principio su reconocimiento a aquellas iniciativas privadas surgidas en el campo de la educación popular, las cuales habían permitido al Estado eximirse de tan onerosas tareas y centrarse en otras cuestiones que se consideraban de mayor importancia, tales como la valorización de la escuela de estudios clásicos, considerada el caldo de cultivo para la formación de la nueva clase dirigente de la Nación.

En este contexto se enmarcaba el propósito de Gentile de valerse, más de lo que se había hecho anteriormente, de la ayuda de la Obra contra el Analfabetismo, orientando su labor en dos direcciones: por un lado, ampliando su acción a todo el territorio nacional y no solo en la Italia centro meridional; por otro, encomendándole la gestión disciplinaria y didáctica de todas aquellas escuelas de educación primaria de bajo rendimiento de cuyo mal funcionamiento había quejas. Las intenciones del ministro, y de su principal colaborador en este campo, Giuseppe Lombardo Radice, fueron llevadas a la práctica por medio del Decreto 2410 del 31 de octubre de 1923. En virtud del mismo, se eliminaba la antigua distinción entre escuelas urbanas y escuelas rurales basada en el número de habitantes y en los ingresos fiscales de los ayuntamientos, subdividiendo las escuelas en dos grupos, escuelas «clasificadas» y escuelas «no clasificadas», en función del número de individuos sujetos a la obligada asistencia. Las primeras eran gestionadas por la administración educativa y los ayuntamientos, se abrían en las capitales de municipio y en las entidades locales menores que tuvieran más de cuarenta niños sujetos a la obligación de asistencia, y se caracterizaban por la presencia de las etapas superior e inferior. A su vez, las «no clasificadas» se subdividían 
en «escuelas provisionales» $\mathrm{y}$ «escuelas subvencionadas». Las primeras estaban en manos de entidades o asociaciones culturales delegadas específicamente por el Estado, se abrían en lugares donde había un número de estudiantes no superior a cuarenta ni inferior a quince, y disponían solo de etapa inferior. Las segundas, sin embargo, podían ser gestionadas por asociaciones privadas, previa autorización del superintendente, y eran parcialmente subvencionadas por el Estado. Junto a la nueva clasificación de las escuelas, el antes mencionado decreto procedía a modificar parcialmente también la Obra contra el Analfabetismo, que cambiaba su nombre a Comité contra el Analfabetismo (Comitato contro l'Analfabetismo). Otro aspecto relacionado con el nacimiento del Comité contra el Analfabetismo era de tipo económico. De hecho, proceder con las denominadas «desclasificaciones», es decir, con la concesión del estatus de escuelas no clasificadas, significaba un ahorro de alrededor de un tercio.

Por lo que respecta por último al aspecto didáctico, es oportuno señalar que la calidad de la enseñanza en las escuelas provisionales o subvencionadas existentes en el campo era mucho menor que la de las escuelas urbanas. Las dos limitaciones principales eran la agrupación bajo un solo maestro - en una sola clase- de niños de primero, segundo y tercer curso de educación primaria y el hecho de que el horario era reducido. Además, quienes enseñaban en las escuelas rurales eran por lo general maestros primerizos, recién salidos del instituto magisterial y carentes de cualquier experiencia profesional, así como, a menudo, procedentes de centros urbanos y, por lo tanto, menos propensos a la vida en el campo. Fue así como, para consagrar también en el ámbito didáctico el diferente estatus de las escuelas rurales, fueron aprobados el 21 enero de 1924, bajo el ministerio de Gentile, los planes de estudio pormenorizados para este tipo de escuelas. En esencia, resultaban más ágiles y simplificados que los de las escuelas urbanas: el programa de enseñanza de Lengua, por ejemplo, se dividía entre el primer y segundo curso, mientras que en el tercero el maestro estaba autorizado a pasar por alto la redacción mensual y el diario de vida escolar, y el espacio asignado al dibujo libre se reducía, así como el canto, cuya enseñanza era recomendada durante las pausas entre una clase y otra. ${ }^{17}$

${ }^{17}$ Giorgio Gabrielli, «I programmi per le scuole rurali», I diritti della scuola, 24 (6 de abril de 1924): 369-371. 
Si el proceso de fascistización de la escuela de educación primaria italiana estaba dando sus frutos más evidentes en las escuelas urbanas y en las rurales gestionadas directamente por el Estado, muy diferente era la situación en las escuelas rurales (no clasificadas) gestionadas por las entidades delegadas. Estas, de hecho, eran sometidas a un menor control por parte de los órganos de supervisión tradicionales, aun cuando las entidades que gestionaban dichos órganos tuvieran la obligación de presentar informes periódicos de su actividad a las autoridades estatales, y el consejo directivo del Comité contra el Analfabetismo estuviera compuesto mayoritariamente por funcionarios ministeriales. Pero, en cuestión de poco tiempo las cosas comenzaron a cambiar y el opresivo clima intolerante que caracterizaba la vida política italiana empezó a hacerse notar también en el mundo de las entidades delegadas. Fue en particular con la llegada al ministerio de Pedro Fedele — que sucedió en enero de 1925 a Casati-cuando los controles sobre las actividades de dichas entidades y de las personas que formaban parte de ellas se intensificaron hasta el punto de resultar insoportables. Gran motivo de preocupación constituyeron, principalmente, los cambios que por decreto ley se introdujeron en el Comité contra el Analfabetismo, cambios que conducirían a un incremento de los controles por parte del gobierno y, al mismo tiempo, a una menor libertad de acción para las asociaciones delegadas. Se trataba de una perspectiva que generó alarma entre quienes consideraban indispensable una cierta autonomía organizativa, financiera y cultural de sus asociaciones, temiendo la intrusión del Estado y del fascismo. Una de las asociaciones más sensibles al tema del mantenimiento de su propia autonomía fue la Animi, también por la presencia en su consejo directivo de personalidades con una clara orientación antifascista como Umberto Zanotti Bianco, Benedetto Croce, Gaetano Salvemini y Giuseppe Lombardo Radice. No sería por cierto casual que precisamente esta fuera la que protestó más enérgicamente en contra de tal propuesta, planteándose como reacción la posibilidad de renunciar a la delegación estatal y, por tanto, a la gestión de las escuelas rurales durante el siguiente año escolar 1925-1926.

El pulso que en el verano de 1925 enfrentó al consejo directivo de la Animi con el gobierno evidenció la completa incapacidad del primero para resistirse a las presiones políticas del segundo. De hecho, se llegó a un punto en el que el ministro dictó una orden categórica: o la asociación 
mantenía su delegación o su junta sería disuelta e intervenida. Alternando amenazas con momentos de mayor diálogo, finalmente el gobierno fue capaz de imponer su línea: no solo no se llevaron a cabo los cambios deseados, sino que con la nueva ley se suprimía incluso el Comité contra el Analfabetismo y se reducía la autonomía de gestión y administrativa de las entidades delegadas, que terminaron bajo la supervisión directa del ministerio. La batalla librada por los consejeros de la Animi se concluía así de la peor de las maneras.

No obstante, y a este respecto, hay que precisar que el decreto firmado por Fedele introducía novedades, no solo con respecto a las asociaciones, sino también en relación con la definición de escuela rural: la vieja denominación de «escuelas provisionales», adoptada por la reforma Gentile, se sustituyó por la de «escuelas no clasificadas». En ellas se incluían todas aquellas establecidas allí donde un solo maestro pudiera satisfacer las necesidades educativas de la población, distribuida dicha población en un área con un radio de dos kilómetros, y un número de alumnos asistentes no inferior a quince ni superior a sesenta. La gestión de dichas escuelas «no clasificadas» fue confiada por delegación del ministerio - y no ya por el suprimido Comité contra el Analfabetismo- a diez entidades, entre ellas siete que hasta entonces habían gestionado las escuelas provisionales: la Sociedad Humanitaria (Società Umanitaria) obtuvo la delegación para Véneto y Venecia Julia; el Grupo de Acción para las Escuelas del Pueblo (Gruppo d'Azione per le Scuole del Popolo) para Lombardía; el Comité Ligur para la Educación del Pueblo (Comitato Ligure per l'Educazione del Popolo) para Liguria; el Ente Nacional de Cultura (Ente Nazionale di Cultura) para Toscana y Emilia; Escuelas para los Agricultores del Agro Romano y las Lagunas Pontinas (Scuole per gli Agricoltori dell'Agro Romano e delle Paludi Pontine) para Lacio, Umbría, Abruzos y Marcas; el Consorcio Nacional de Emigración y Trabajo (Consorzio Nazionale di Emigrazione e Lavoro) para Campania y Molise y, por último, la Animi para Basilicata, Calabria, Sicilia y Cerdeña. A las asociaciones ya existentes se añadieron tres más: el Ente Pugliés de Cultura (Ente Pugliese di Cultura) que, a pesar de haber nacido en 1923 con el fin de organizar clases nocturnas para los trabajadores en la provincia de Bari, ahora también recibía la delegación para la gestión de las escuelas rurales, así como las nocturnas y las festivas, en Apulia por voluntad del fascismo local, restando esta tarea a la Sociedad Humanitaria de Milán; 
a la Organización Nacional de Asistencia para la Italia Redimida (Opera Nazionale di Assistenza per l'Italia Redenta), de ahora en adelante llamada Onair —asociación fundada en 1919 bajo el patrocinio de la duquesa de Aosta para proporcionar asistencia moral y material a las poblaciones de las nuevas provincias anexionadas al Reino después de la primera Guerra Mundial, que hasta entonces había gestionado principalmente guarderías y proporcionado asistencia sanitaria-, se le asignó la delegación para Venecia Julia y Trentino (Venezia Tridentina); ${ }^{18}$ por último, el Grupo de Acción para las Escuelas Rurales en Piamonte (Gruppo d'Azione per le Scuole Rurali per il Piemonte), asociación fundada en Turín en marzo de 1923 por iniciativa de Giovanni Vidari con el objetivo de asistir en su acción educativa a los maestros de las escuelas rurales de Piamonte y de los valles alpinos "para animarles y ayudarles en su acción educativa», establecer una biblioteca móvil para los maestros y facilitarles el suministro de material didáctico, recibió la delegación para Piamonte y los valles alpinos. ${ }^{19}$

Mientras tanto, el proceso de «desclasificación» de las escuelas rurales, iniciado bajo el ministerio Gentile, experimentó una fuerte aceleración en gran medida en virtud de las nuevas disposiciones. Si en el año escolar 1926-27 las escuelas no clasificadas en toda Italia ascendían a 3.479 , al año siguiente a estas se les añadieron otras 1.264 escuelas, de las cuales la gran mayoría (nada menos que 1.164) habían sido hasta entonces escuelas clasificadas y solo una mínima parte (100) eran de reciente creación. $^{20}$

Después de haber reorganizado, de la forma en que se acaba de describir, la rama de la educación rural, el régimen llevó adelante en la segunda mitad de los años veinte su labor de penetración dentro de las entidades delegadas, debilitando su acción o alejando de ellas a los dirigentes con ideas no fascistas o bien sustituyéndolos por otros organismos de clara

\footnotetext{
${ }^{18}$ Giuseppe Scarascia, «L'Opera Nazionale di Assistenza all'Italia redenta», Annali dell'istruzione elementare, 1 (enero- febrero de 1926): 31-41.

${ }^{19}$ Informaciones sobre las actividades de la asociación se pueden encontrar en el Gruppo d'azione per le scuole rurali del Piemonte. Relazione sull'opera compiuta negli anni 1923-24, 1924-25 (Torino: Tipografia Roggero \& Tortia, 1925).

${ }^{20}$ Los datos se toman de la Tabla II publicada en Guido Ruberti, «Fondamento e caratteri della scuola elementare attraverso la più recente indagine statistica», Annali dell'istruzione elementare, 1 , (enero-febrero de 1928), 43.
} 
confianza política. Junto con la expulsión de personas no gratas, se inició una campaña de deslegitimación de las entidades delegadas por parte de los sectores más extremistas del fascismo — como la Asociación Nacional de Maestros Fascistas (Associazione Nazionale Insegnanti Fascisti), de ahora en adelante llamada ANIF- que no compartían la decisión tomada por el gobierno de confiar a organismos privados la gestión de un sector importante de la educación primaria. El 15 de abril de 1927, por ejemplo, en La Escuela Fascista (La scuola fascista), órgano de ANIF, se publicó un artículo en el que se criticaba el excesivo poder entregado a las entidades delegadas, y se reafirmaba el principio de que «el Estado fascista no debería delegar en nadie, cualesquiera que sean sus méritos, lo que es tarea suya» ya que la "escuela de educación primaria es su territorio privilegiado, sin derecho a condominio». ${ }^{21}$

A estas polémicas respondía La Nueva Escuela Italiana (La Nuova Scuola Italiana), revista relacionada con el Ente Nacional de Cultura, última frontera desde un punto de vista periodístico para la defensa de las entidades, junto con El Grupo de Acción (Il Gruppo d'Azione), boletín del Grupo de Acción de las Escuelas del Pueblo de Milán (Gruppo di Azione delle Scuole del Popolo di Milano). En una serie de artículos publicados en la primavera de 1928 la revista recordaba cómo las asociaciones habían nacido con la Reforma Gentile, que fue definida por Mussolini como «la más fascista de todas las reformas», una circunstancia que hacía incomprensibles las críticas de los que blandían el argumento según el cual estas no estarían actuando por voluntad expresa del Estado, o peor, que manifestaban simpatías antifascistas. ${ }^{22}$

No menos fácil fue la vida para la Animi. A principios de 1926 se le involucraba en una agria disputa entre Lombardo Radice y algunos colaboradores de Gentile, ya alineados en posiciones fascistas, a causa de los repetidos artículos críticos hacia ANIF escritos por el pedagogo siciliano en La Educación Nacional (L'Educazione Nazionale). Quien contestó a Lombardo Radice fue La Educación Política (L'Educazione Politica), revista dirigida por el mismo Gentile, en un editorial firmado y titulado $L a$ redacción (La redazione), en el que al educador se le acusaba de «antifascismo académico» y de ser un «campeón del moralismo aventino». Para

21 «Sclassificazione di scuole», La scuola fascista, (15 de abril de 1927): 6.

22 «La scuola rurale unica mista», La nuova scuola italiana, 25 (18 de marzo de 1928): 754-756. 
terminar el artículo se sacaba a relucir la Animi, y se le acusaba de un «insípido y ridículo laicismo». ${ }^{23}$

Pero el accidente más grave se produjo — según recuerda Zanotti Bianco- en marzo de 1928, cuando el ministro envió una carta al presidente de la Animi que condicionaba su labor. Tal y como se enteraron después por una circular ministerial, la reunión tenía un propósito meramente político: «la fascistización de las escuelas rurales no clasificadas y la formación a implantar de los «Balilla» y de las Jóvenes Italianas (Giovani Italiane)», y la solicitud a los organismos de su «ferviente e incondicional labor para la aplicación íntegra de las directrices marcadas al respecto por el Muy Honorable Ministro en obediencia a la precisa voluntad del Duce». ${ }^{24}$ Esta vez la renuncia de la Animi a la delegación fue definitiva: habiéndose liberado de una carga que ya no podía soportar bajo tales condiciones, la asociación podía ahora centrarse en otras cuestiones, en particular en la gestión de los jardines de infancia. El régimen no dudó en aprovecharse de esta situación para llevar a cabo ese proceso de fascistización de la escuela rural siempre anhelado: por Decreto 2176 del 6 de septiembre de 1928 se estableció la avocación de las escuelas no clasificadas de Sicilia y Calabria a la Obra Nacional Balilla (Opera Nazionale Balilla - - de ahora en adelante llamada ONB—, y de este mismo tipo de escuelas en Cerdeña al Ente de Cultura y Educación de Cerdeña (Ente di Cultura e di Educazione della Sardegna). ${ }^{25}$ La ONB vio así como se le entregaba en las dos regiones meridionales un conjunto de 477 escuelas que adoptaron la nueva denominación de Escuelas Rurales Obra Nacional Balilla (Scuole Rurali Opera Nazionale Balilla), además de un elevado número de escuelas nocturnas. ${ }^{26} \mathrm{Al}$ año siguiente heredó también la gestión de las escuelas rurales de Cerdeña. La avocación a la ONB de las escuelas rurales de Calabria, Sicilia y Cerdeña supuso un cambio relevante, en especial por razones políticas, ya que esta experiencia constituyó una especie de laboratorio para la creación de la nueva escuela fascista.

\footnotetext{
${ }^{23}$ Carta de Piacentini a Gentile, 5 de marzo de 1926, Correspondencia, carpeta 4454, Archivo «Giovanni Gentile».

${ }^{24}$ Umberto Zanotti Bianco, L'Associazione nazionale per gli interessi del Mezzogiorno d'Italia nei suoi primi cinquant'anni di vita (Roma: Collezione Meridionale, 1960), 54-55.

${ }^{25}$ Bollettino Ufficiale del Ministero della Pubblica Istruzione, 42, (16 de octubre de 1928): 3384-3385.

${ }^{26}$ «Le Scuole dell'Opera Nazionale Balilla nell'anno 1928-29», Annali dell'istruzione elementare, 5, (octubre de 1929): 94-95.
} 
Con el ascenso al ministerio de Balbino Giuliano, que tuvo lugar en septiembre de 1929, las entidades delegadas parecían encontrar una protección bajo cuya ala podían protegerse de los ataques de aquellos sectores del fascismo más extremistas que exigían el traspaso de todas las escuelas rurales al Estado o a la Opera Balilla. El nuevo ministro, que se consideraba discípulo de Gentile, se preocupaba sin duda por la suerte de las entidades, teniendo en cuenta que desde junio de 1925 hasta ese momento había ocupado el cargo de presidente del Ente Nacional de Cultura, asociación que gestionaba las escuelas rurales no clasificadas en Toscana y Romaña. Una primera oportunidad de demostrarlo fue su intervención en la Cámara, a finales de marzo de 1930, durante la cual dos diputados exigieron el traspaso de todas las escuelas rurales a la Opera Ballila, uniformando el resto del país con lo que se había hecho hasta entonces en Calabria, Sicilia y Cerdeña. ${ }^{27}$ Fue, sobre todo, el diputado Francesco Bascone - uno de los fundadores de la Corporación de la Escuela (Corporazione della Scuola) — quien declaró que el Estado no podía renunciar a la formación de una parte considerable de los jóvenes, después de haber afirmado el principio según el cual la educación era una función eminentemente estatal. En 1930, el asalto a las escuelas gestionadas por las entidades fracasó, pero en la práctica solo se pospuso. A cambio, la Opera Balilla había conseguido extender sus tentáculos también sobre las escuelas rurales (tanto las escuelas rurales administradas por el Estado, como las no clasificadas gestionadas por las entidades) a través de otros tres instrumentos: la afiliación de los alumnos a quienes se les concedía el título de Balilla y de Pequeña Italiana, la asignación educación física a un instructor de la ONB, y la politización de la enseñanza con la celebración del Duce y del Régimen. ${ }^{28}$ El nivel de fascistización de la escuela rural, por tanto, no debía ser tan diferente en comparación con el que caracterizaba al resto de las escuelas de educación primaria.

Sin embargo, esto ya no era suficiente a principios de los años treinta. El cerco a la escuela rural se hizo más intenso, de hecho, con la llegada al poder en julio de 1932 del nuevo ministro, Francesco Ercole. Bueno es recordar que él había sido uno de los signatarios del orden del día

\footnotetext{
${ }^{27}$ «La scuola elementare nella discussione del Bilancio dell'Educazione Nazionale», La nuova scuola italiana, 28, (13 de abril de 1930): 7.

28 Jürgen Charnitzky, Fascismo e scuola. La politica scolastica del regime fascista, 1922-1943 (Firenze: La Nuova Italia, 1996), 332.
} 
presentado por el diputado Bascone pocos meses antes en la Cámara, en el que se pedía de nuevo la avocación de las escuelas no clasificadas al Estado. Su posición con respecto a este tema, por lo tanto, se conocía hacía tiempo y no hará falta mucho para ver qué acciones implantaría. A partir de los primeros meses de 1933 las entidades delegadas quedaban, por orden del ministro, sujetas a una auditoría contable y administrativa, presentada como un acto informativo, pero en realidad orientada a debilitar las entidades y a intimidar a sus dirigentes. Sin embargo, dicha inspección no dio los resultados esperados. ${ }^{29}$

El año escolar 1933-1934 comenzaba, por tanto, con muchas sombras para las entidades delegadas, al mismo tiempo que se sucedían las voces por los pasillos ministeriales acerca de inminentes medidas del gobierno. Se llegó, pues, a mediados de noviembre, cuando la noticia que muchos esperaban apareció en los periódicos. A la prensa se le había remitido un "comunicado extraoficial» en el que se preanunciaba que, a partir del siguiente año escolar 1934-1935, se quitaría la gestión de las escuelas rurales únicas operativas en los territorios de Génova, Turín, Milán, Venecia, Bolonia, Florencia, Nápoles y Campobasso a las organizaciones que hasta la fecha la habían llevado, y se confiaría a la Opera Balilla, mientras que las escuelas rurales únicas de la zona de Trieste se entregarían a la Onair. ${ }^{30}$ De las organizaciones activas hasta ese momento permanecerían, por tanto, en funcionamiento — además de la Onair y de la ONB-, solo el Ente Pugliés de Cultura y las Escuelas para los Campesinos del Agro Romano.

La noticia de la retirada de la delegación a la mayoría de las asociaciones anticipada en noviembre de 1933 se convirtió en realidad con el Decreto de 15 de junio de 1934, que establecía la transferencia a la Opera Balilla de las escuelas rurales únicas de Lombardía, Piamonte, Veneto, Liguria, Emilia, Toscana, Campania y Molise, administradas hasta entonces por entidades delegadas. ${ }^{31}$ Permanecían en funcionamiento las

\footnotetext{
${ }^{29}$ Alessandro Marcucci, La scuola di Giovanni Cena: Per la scuola popolare (Torino: G.B. Paravia e C., 1948), 219.

30 «Nelle scuole rurali delle Associazioni delegate», I diritti della scuola, 7 (19 de noviembre de 1933): 103-104; «La gestione delle scuole uniche rurali», La nuova scuola italiana, 9 (19 de noviembre de 1933): 6.

${ }^{31}$ Renato Marzolo, «La scuola rurale dell'Opera Balilla», Annali dell'istruzione elementare, 1 (febrero de 1935): 19.
} 
escuelas del Agro Romano en Lacio, Abruzos, Marcas y Umbría, mientras que el Ente Pugliés de Cultura pudo continuar funcionando en Apulia y Lucania. Por último, la Onair pudo mantener la delegación en Venecia Tridentina, a la que añadió Venecia Julia. ${ }^{32}$

Que esta situación no duraría mucho tiempo estaba claro, incluso para las personas directamente afectadas. Como se temía, el golpe de gracia llegó con el Decreto del 25 de febrero de 1935, por medio del cual el nuevo ministro de Educación Nacional, De Vecchi Di Val Cismon, dispuso para el año escolar 1935-1936 la transferencia a la ONB de las escuelas no clasificadas presentes en el territorio de Roma, L'Aquila, Ancona, Perugia, Bari y Potenza. En otras palabras, se retiraba de este modo la delegación a las Escuelas del Agro Romano y al Ente Pugliés de Cultura. ${ }^{33}$ Incluso, desde un punto de vista formal, se quiso sancionar la nueva condición jurídica de las escuelas no clasificadas. Así, en junio de 1935, estas adoptaron el nombre de «escuelas rurales». ${ }^{34}$

Absorbida por la Opera Balilla, la escuela rural se convirtió en el terreno privilegiado en el que experimentar con la formación del nuevo campesino fascista, cuyas virtudes tenían que ser la sobriedad, la laboriosidad, el orgullo y el amor por la patria. El campo era el lugar en el que vivir, con un alto sentido de la dignidad, la verdadera vida sencilla y frugal, en contraposición al lujo y a las comodidades ostentadas en las ciudades corruptoras del alma del agricultor, de acuerdo con la política «ruralizadora» del Régimen. Los jóvenes campesinos podían por fin educarse en un ambiente sano, en el culto a la Patria y en el amor por su propia tierra, por lo que el viejo lema «libro y mosquete» — que daba la idea de un militante dedicado al estudio, al ejercicio físico y al uso de las armas para defender la revolución-, se podía sustituir, en opinión del senador Nicola Pende, por otro mucho más apropiado para indicar la «verdadera regeneración integral de la escuela fascista: libro, mosquete y azada». ${ }^{35}$

Con el advenimiento de Giuseppe Bottai al Ministerio en noviembre de 1936, el proceso de fascistización de la escuela rural alcanzaba su pun-

\footnotetext{
32 Bollettino Ufficiale del Ministero dell'Educazione Nazionale, 29 (17 de julio de 1934): 1506-1507.

${ }^{33}$ Bollettino Ufficiale del Ministero dell'Educazione Nazionale, 13 (26 de marzo de 1935): 893-894.

${ }^{34}$ Charnitzky, Fascismo e scuola, 435.

${ }^{35}$ Nicola Pende, «La scuola fascista nella sua fase corporativa, imperiale e biologica», Rivista pedagogica, 3 (1937): 256.
} 
to más alto. El nuevo responsable de la Educación Nacional, de hecho, prosiguió por el camino iniciado por sus predecesores destinado a intensificar el control sobre la educación primaria en el campo y a conferirle a esta una "orientación rural» más pronunciada, introduciendo esta rama de la enseñanza en su forma más compleja y estructurada representada por la Carta de la escuela (Carta della Scuola), que cambiaría todo el edificio educativo nacional después de la Reforma Gentile. ${ }^{36} \mathrm{El}$ acto más importante, desde este punto de vista, fue la avocación al Estado de casi todas las escuelas rurales en Italia, establecida por el Decreto 1771 del 14 de octubre de 1938. De acuerdo con esta disposición, a partir del 1 de enero de 1939 las escuelas, antes gestionadas por la Opera Balilla —sustituida en octubre de 1937 por la Juventud Italiana del Littorio (Gioventù Italiana del Littorio) - , pasaron directamente bajo la autoridad del Ministerio, a excepción temporal de las pocas escuelas que le quedaban al Ente Escuelas para los Campesinos del Agro Romano, a la Onair y a las entidades de mejora agraria. ${ }^{37}$

La definición de escuela rural también cambió: ahora se consideraban como tales las escuelas de educación primaria de las capitales de municipios, entidades geográficas menores o aldeas, con un número de niños en edad de obligada escolarización no superior a 250 ni inferior a 20, y cuando se tratara de lugares habitados por una población dedicada principalmente a la agricultura. Para satisfacer los deseos de los maestros, que desde tiempo atrás se quejaban de la desigualdad de trato con respecto de los colegas de las escuelas urbanas, Bottai abrió el camino para una mejora de la perspectiva laboral que les hizo entrever mejores condiciones salariales. Desde un punto de vista cuantitativo, las escuelas rurales a fecha del 1 de enero de 1939 eran 7.170, en el año 1939-1940 subieron a 7.529 y en 1940-1941 a 8.129. En esos años, fue posible ampliar, en muchos casos, la etapa de primaria, con el establecimiento del cuarto $\mathrm{y}$, a veces, el quinto curso.

Mientras tanto, con la nueva reorganización del sistema educativo previsto por la Carta de la escuela (1939), el mismo concepto de escuela rural evolucionó con respecto a lo redactado por la pedagogía idealista y a lo llevado a la práctica por la Reforma Gentile. De hecho, la reforma,

${ }^{36}$ Charnitzky, Fascismo e scuola, 440-469.

${ }^{37}$ Ministero della Educazione Nazionale, Scuole Rurali (Roma, 1940), 5. 
con el objetivo de introducir en Italia la llamada «escuela del trabajo» - de acuerdo con la IX declaración de la Carta de la escuela, que preveía la preparación al trabajo manual de los niños de nueve a once años de edad-, empujó la escuela rural a definirse en sentido práctico siendo su finalidad la de formar al buen y laborioso trabajador del campo. Sin embargo, la reforma tenía que ser ante todo una reforma espiritual, incluso antes de abordar el aspecto económico y productivo. Tenía que cambiar la mentalidad y la cultura con el fin de construir un sistema de valores para los jóvenes campesinos que pusiera en el centro el campo, el trabajo agrícola y su ética.

En una Italia ya exhausta por tres largos años de guerra, se establecía finalmente mediante la Ley del 31 de mayo de 1943 que las escuelas rurales todavía gestionadas por la Onair y las Escuelas para los Campesinos del Agro Romano tendrían que pasar al Estado a más tardar el 30 de septiembre de ese año. El armisticio del 8 de septiembre y la escalada de los acontecimientos bélicos con la ocupación alemana de Italia terminarían por hacer innecesaria cualquier medida legislativa que apuntara a la consagración de uno de los principios más preciados del fascismo, aquel según el cual solo el Estado ético tendría que haberse ocupado de la educación de sus jóvenes, también de aquellos del campo, con el fin de forjar el hombre nuevo deseado por Mussolini.

\section{Nota sobre el autor}

Luca Montecchi es investigador de Historia de la Educación en la Universidad de Macerata. Profesor de Historia de la Educación e Instituciones Escolares de la Universidad de Perugia en el año académico 2016/2017, es autor de algunos estudios sobre la historia de la educación en el mundo campesino italiano entre los siglos XIX y XX.

\section{REFERENCIAS}

Bertoni Jovine, Dina. Storia della scuola popolare in Italia. Torino: Einaudi, 1953. BONETTA, Gaetano. "Insegnare la cultura materiale. Istruzione agraria e lavoro manuale nell'Italia del XIX secolo». In Storia delle Istituzioni educative in Italia tra Otto e Novecento, edited by Lucia Romaniello, 153-164. Milano: Amici del Museo del Risorgimento, 1996. 
CATARsi, Enzo. Storia dei programmi della scuola elementare, 1860-1985. Firenze: La Nuova Italia, 1990.

CHARNITZKY, Jürgen. Fascismo e scuola. La politica scolastica del regime fascista, 1922-1943. Firenze: La Nuova Italia, 1996.

CHIosso, Giorgio. L'educazione nazionale da Giolitti al primo dopoguerra. Brescia: La Scuola, 1983.

InZERILlo, Giuseppe. Storia della politica scolastica in Italia. Da Casati a Gentile. Roma: Riuniti, 1974.

LEVRA, Umberto. Il colpo di stato della borghesia. La crisi politica di fine secolo e l'età giolittiana. Torino: UTET, 1982.

Marcucci, Alessandro. La scuola di Giovanni Cena: Per la scuola popolare. Torino: G.B. Faravia, 1948.

Montecchi, Luca. I contadini a scuola. La scuola rurale in Italia dall'Unità alla caduta del fascismo. Macerata: Eum, 2015.

ZANOtTI BIANCO, Umberto. L'Associazione nazionale per gli interessi del Mezzogiorno d'Italia nei suoi primi cinquant'anni di vita. Roma: Collezione Meridionale, 1960 . 